\title{
(6) OPEN ACCESS \\ Why do people present late with advanced glaucoma? A qualitative interview study
}

\author{
Maria Prior, ${ }^{1}$ Jill J Francis, ${ }^{2}$ Augusto Azuara-Blanco, ${ }^{3}$ Nitin Anand ${ }^{4}$ Jennifer M Burr, ${ }^{5}$ \\ for the Glaucoma screening Platform Study group
}

${ }^{1}$ Health Services Research Unit University of Aberdeen, Aberdeen, UK

${ }^{2}$ School of Health Sciences, City University London, London, UK

${ }^{3}$ School of Medicine, Dentistry and Biomedical Sciences, Queen's University Belfast, Belfast, UK

${ }^{4}$ Department of Ophthalmology, Huddersfield Royal Infirmary, Huddersfield, UK

${ }^{5}$ School of Medicine, University of St Andrews, St Andrews, Fife, UK

\section{Correspondence to} Dr Maria Prior, Health Services Research Unit, University of Aberdeen, Health Sciences Building, Foresterhill, Aberdeen AB25 2ZD, UK: m.e.prior@abdn.ac.uk

Received 4 June 2013 Revised 11 July 2013 Accepted 25 August 2013 Published Online First 24 September 2013

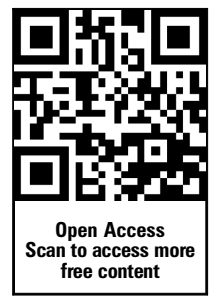

To cite: Prior $\mathrm{M}$, Francis JJ, Azuara-Blanco $\mathrm{A}$, et al. Br J Ophthalmol 2013;97 1574-1578.

\section{ABSTRACT}

Objective To explore the presentation behaviours and pathways to detection of adults who first presented to UK hospital eye services with severe glaucoma.

Design Semistructured interviews, based on models of diagnostic delay, to obtain a descriptive self-reported account of when and how participants' glaucoma was detected.

Results 11 patients participated (five in Aberdeen, six in Huddersfield). Four participants reported that the optometry appointment at which their glaucoma was detected was their first ever eye test or their first for over 10 years. Seven participants reported attending regular routine optometrist appointments. Their self-reported experiences and pathways to detection describe a variety of missed detection opportunities and delayed referral and treatment.

Conclusions The qualitative data suggest that late detection of glaucoma can result from delays at the patient level but, although based on a small sample, delays also occurred at the healthcare provider (system) level both in terms of accuracy of case detection and effective referral. We suggest that current attempts to address the significant burden of over-referral of glaucoma suspects to hospital eye services (a large proportion of which are false positives) must also focus on the issue of false negatives and on reducing missed detection and service delays.

\section{INTRODUCTION}

Glaucoma is a condition where early detection and thus access to treatment is associated with better outcomes. ${ }^{1-3}$ Studies have identified delays attributable to patients' late presentation for eye tests, ${ }^{4}$ such delays being associated with risk factors such as socioeconomic deprivation, African-Caribbean ethnicity and older age. ${ }^{4-6}$ However, little is known about the experiences of patients with severe glaucoma at diagnosis, for example, their presentation behaviours and pathways to detection. Greater understanding is essential for the development of successful interventions to optimise timely detection, diagnosis and treatment.

Existing models of 'diagnostic delay' describe decisional and behavioural processes prior to treatment. These include delay attributable to patients (eg, delay in seeking care) and diagnostic and treatment delay attributable to healthcare providers (at the individual practitioner level or at the service level). ${ }^{7-9}$ Figure 1 illustrates the stages at which diagnostic delay of glaucoma may occur, the nature of the delay and the behaviour required (by the patient or healthcare provider) in order to access appropriate treatment.
In this qualitative study, we explored accounts of when and how glaucoma was detected in adults who first presented to hospital eye services with severe glaucoma.

\section{METHODS}

\section{Sampling and recruitment}

We sought narratives from adults who first presented to hospital eye care services (in Aberdeen or Huddersfield, UK) with glaucomatous visual field loss of $-20 \mathrm{~dB}$, or worse, in at least one eye, or bilateral loss of $\geq-12 \mathrm{~dB}$ in both eyes. Consecutive patients meeting this eligibility criterion were identified from two glaucoma outpatient clinic lists (Aberdeen, Huddersfield) by clinical ophthalmologists and sent an information leaflet and a letter of invitation to participate in the study (signed by the clinician). The purposive sample was not intended to be representative or to estimate the distribution of views across the population of people presenting with late stage glaucoma. Its aim was to generate insight into the glaucoma patients' experiences and presenting behaviours.

\section{Data collection}

The study was approved by North of Scotland research ethics committee (Ref: 09/S0801/68). Semistructured face-to-face interviews were conducted, between March and November 2009, using a topic guide based on models of patient and provider delay. $^{7-10}$

We obtained a descriptive account, in the participant's own words, of when and how their glaucoma was detected. In addition, we collected self-reported demographic and medical information (eg, ethnicity, family history of glaucoma) to describe our purposive sample. Formal consent was obtained. Interviews were audio-recorded, transcribed verbatim and anonymised using a unique participant identifier (P01-P11) and all other identifiable information removed (eg, ophthalmologists' names).

\section{Data management and analysis}

We used a directed content analysis approach based on existing models of diagnostic delay ${ }^{7-9}$ to identify the behaviours and experiences that led to detection of the participants' glaucoma (detection pathways). ${ }^{11}$ Directed content analysis involves the use of existing theory or prior research to provide initial coding categories. However, this did not preclude the emergence of new themes. One researcher (MP) worked systematically through all transcripts and created charts to summarise issues that related to participants' presentation 


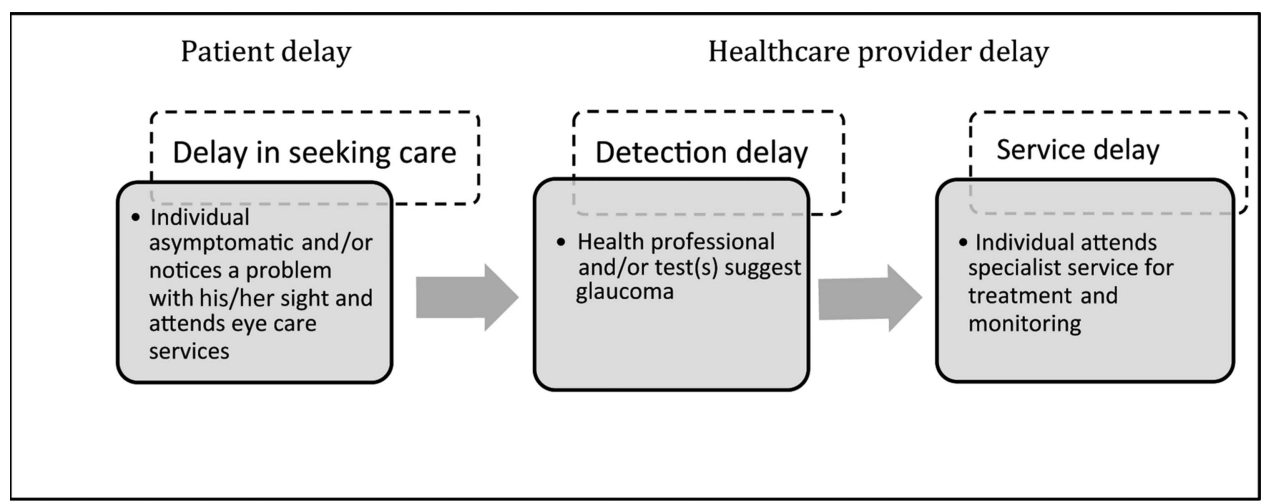

Figure 1 Detection and treatment pathway for glaucoma and stages of diagnostic delay. Adapted from refs. 7-10

behaviours, experiences and pathways to detection. For reliability, another researcher $(\mathrm{JB})$ read and coded a sample of transcripts. The findings were consistent.

\section{RESULTS}

\section{Sample characteristics}

Fourteen patients were invited to participate in the study (Aberdeen: eight; Huddersfield: six). Of these, 11 expressed an interest in taking part (Aberdeen: five; Huddersfield: six). All 11 were interviewed. No further contact was made with the other three patients. The 11 participants included seven women and four men. Table 1 presents their self-reported characteristics. A summary of each participant's self-reported case history and detection pathway is presented in table 2 .

\section{Presentation behaviours and detection pathways}

Participants' accounts of when and how their glaucoma was detected were diverse. Most described the detection and subsequent diagnosis of advanced glaucoma as memorable and often distressing events, but were keen to have the opportunity to discuss and share.

Four participants reported that the optometry appointment at which their glaucoma was detected was their first ever eye test or their first for over 10 years. Seven participants reported attending regular routine optometrist appointments. Their descriptions of experiences and pathways to detection included a variety of missed detection opportunities, which in some cases were combined with delays in accessing specialist ophthalmology services for treatment and monitoring. Quotes from interviews are presented below to illustrate the study findings.

\section{Patient delay}

Delay in seeking care

Participants had difficulty recalling how long it took for them to notice a problem with their eyesight. Nevertheless, accounts from all four patients who did not regularly attend optometry services included reports of noticeable changes in vision being the stimulus to making a non-routine optometry appointment that led to their glaucoma being detected and immediate referral to hospital eye services.

I started having blurry...running eyes...I noticed occasionally it would be cloudy...I was in [supermarket] one day so I just went to the optician and said I need glasses, so when the guy test my eye...they referred me up to the eye clinic. (P01)

I went to the optician thinking I was needing glasses for the TV... I never wore glasses in my life...I thought well I'm getting on in age, I thought I was needing reading glasses. (P05)

For one participant, it was the work supervisor rather than the participant who first noticed a problem and arranged an optometrist appointment.

[Supervisor suggested] I go for an eye test as there's something wrong. I was shocked, I said I can see properly...I don't think I need an eye test, I don't use glasses... my Supervisor called the optician in the [shopping] centre...he made an appointment. (P03)

Table 1 Participants' self-reported characteristics

\begin{tabular}{|c|c|c|c|c|c|}
\hline ID & Gender, year of glaucoma diagnosis, eyes affected & Age group* (years) & Ethnic group* & Myopia* & Family history of glaucoma* \\
\hline 01 & Man, 2006, one eye & $40-49$ & Black British-Black African & No & No \\
\hline 02 & Man, 2008 , both eyes & $<40$ & Black British-Black African & No & No \\
\hline 03 & Man, 2005 , both eyes & $>70$ & White British & Yes & No \\
\hline 04 & Woman, 2005, both eyes & $>70$ & White British & No & No \\
\hline 05 & Woman, 2004, both eyes & $>70$ & Irish & No & No \\
\hline 06 & Woman, 2003, both eyes & $50-59$ & White British & No & Yes \\
\hline 07 & Woman, 1997, both eyes & $>70$ & White British & No & No \\
\hline 08 & Woman, 2006, both eyes & $60-70$ & White British & No & No \\
\hline 09 & Woman, 2007, both eyes & $>70$ & White British & No & Yes \\
\hline 10 & Man, 2006, 1 eye & $>70$ & White British & Yes & Yes \\
\hline 11 & Woman, 2009, both eyes & $60-70$ & Black British-Black African & Do not know & Do not know \\
\hline
\end{tabular}

${ }^{\text {*}}$ Risk factors for developing the most common form of glaucoma (open-angle glaucoma). 
Table 2 Case histories

\begin{tabular}{|c|c|c|}
\hline ID & Case history and detection pathway & Post-detection pathway to specialist glaucoma services \\
\hline 01 & $\begin{array}{l}\text { Detected on first ever visit to optometrist } \\
\text { Noticing eyes watering and blurry when reading. } \\
\text { Spontaneous decision to go to supermarket optometrist }\end{array}$ & $\begin{array}{l}\text { Patient told by optometrist to phone eye clinic for an appointment } \\
3 \text { months from referral (patient phone call) to eye clinic appointment }\end{array}$ \\
\hline 02 & $\begin{array}{l}\text { Routine eye tests every 1-2 years. Wore glasses. Advanced normal tension } \\
\text { glaucoma detected by 'photograph' at first visit to 'new' optometrist }\end{array}$ & $\begin{array}{l}\text { Patient opted for private first consultation to minimise any opportunity for delay. } \\
\text { Saw private consultant within } 2 \text { weeks. Commenced on ocular hypotensive } \\
\text { therapy (Travoprost) and referred to specialist National Health Service eye clinic }\end{array}$ \\
\hline 03 & $\begin{array}{l}\text { Detected on first ever visit. Patient had not noticed anything was wrong. } \\
\text { Supervisor at work noticed increasing frequency of mistakes at work, suggested }\end{array}$ & $\begin{array}{l}\text { Got letter back from hospital eye clinic within } 2 \text { weeks of optometrist referral. } \\
\text { Appointment was in } 2 \text { months' time }\end{array}$ \\
\hline
\end{tabular}
eye test and booked an appointment

04 Same optometrist for 8 or 9 years. Reporting severe headaches over eyes at last two appointments and problems with glare. Made GP appointment to discuss severity of symptoms GP referred patient to cataract clinic Glaucoma detected at cataract clinic

05 Detected at first optometrist appointment

06 Been to optometrist since age of 8 . Annual visit from age of 40 due to family history of glaucoma

1992: optic disc haemorrhage detected by optometrist. Patient reports no action taken by GP or two optometrists (second optometry opinion sought by patient due to family history)

1994-2003: optic nerve changes noted (increasing cup to disc ratio and between-eye asymmetry in 1999). Patient reporting pain in right eye+headaches +increasing problems with night and then all glare

2000-2002: reporting falls, difficulty crossing road, glare, accommodating to bright/dim light

2003: glaucoma detected by high street optometrist near to her place of work. Appointment made after patient broke her glasses in a fall Advanced glaucoma diagnosed at first appointment with consultant ophthalmologist

07 Routine eye test every 2 years. Same optometrist for many years. He retired, glaucoma detected on next routine visit to his replacement

08 Referred to hospital with high pressures 30 years ago, attended eye clinic, but reports being discharged after a few visits. Routine sight tests every 2-3 years since then

When glaucoma detected it was 3 years since last full eye check

09 Wore glasses for reading, but had not had an eye test for $>10$ years Started to notice things looked a different colour through one eye Optometrist referred patient to cataract clinic

10 Annual eye checks, cataracts and glaucoma detected at routine visit. Detected by an optometrist that patient had seen on previous appointments

11 Detected at annual eye check

With hindsight patient reports pain above the eyes at night (throbbing) for 1 month prior to optometrist appointment, thought it was ear ache

Cataract clinic appointment from GP took 4 months

Cataract clinic initiated ocular hypotensive therapy and referred patient to glaucoma clinic (patient reports having to stay at cataract clinic for $3.5 \mathrm{~h}$ while they tried to reduce pressures)

Patient unsure of exact timescale but 'not long'

15 weeks between detection by optometrist and being seen at glaucoma clinic

Attended hospital eye clinic within 2 days of seeing new optometrist

Patient given letter to take to GP. Patient saw GP the next day

Eye clinic phoned patient, 7 days after she saw GP, asking her to attend eye clinic the following week

\section{Saw GP within 3 days of seeing optometrist}

Patient received letter from hospital with a 'password'. Patient then had to ring hospital to make appointment

Attended hospital appointment (at cataract clinic) within 2-3 weeks, but on first visit was told she was in the wrong place and 'quickly' referred her on to her current glaucoma specialist

Optometrist referred patient to GP (by letter)

GP wrote to patient informing him that an appointment had been made at the hospital. Attended hospital appointment approximately 2 months later

Optometrist referred to GP. GP contacted the hospital

Patient reports attending ophthalmology clinic the day after GP informed by optometrist. Given letter from optometrist/GP to take with her

\section{Healthcare provider delay}

Delays in detection and treatment were suggested to have occurred for two main reasons: a failure to detect glaucoma at routine optometry appointments (detection delay) and referral or waiting time delays following detection (service delay).

\section{Detection delay}

The main 'trigger' for glaucoma detection in participants who reported attending regular routine optometry appointments was a change in optometrist or a General Practitioner (GP) appointment. Participants' narratives provided compelling accounts of detection delay.

It was detected when the optician looking after me at the time retired and somebody came to take his place and said I think that you have glaucoma... it was on my records for quite a number of years ... getting more noticeable. (P07)

It was picked [up] initially when I attend[ed] for a routine [appointment] at my Optician, but he was a new Optician to me...he was the first person who took a photograph of my retina...he wasn't happy with my optic discs... he did visual field tests that confirmed that I had big areas in the upper outer quadrants completely blacked off which I was totally unaware of... he reckoned I'd probably had it for about 4 or 5 years. (P02)

The woman [optometrist] said I had cataract in one eye...next year I went back and the cataract was slightly worse...I had to stop driving, because I couldn't cope with the sun ...so I went to the doctor $[\mathrm{GP}]$...he thought that the cataract was bad so he referred me to the cataract clinic and the doctor there said I'd had it [glaucoma] a long time, it was well advanced...she referred me to the Glaucoma clinic and I was told that I had severe glaucoma in both eyes. (P04)

A few participants described reporting symptoms to optometrists during routine appointments that in retrospect they found 
significant, but which their optometrist did not attribute to glaucoma and which had not led to timely detection.

I've been going to the same Optician for several years, about 8 or 9 years... I said that I'd had headaches, severe headache across my left eye...she said well there's nothing wrong with your eyes...you may have a migraine. (P04)

...from 1994 onwards I was reporting that this eye hurt, it felt like [I] had been thumped... and I kept saying this so every time he [optometrist] was saying to me 'anything to report' my answer to that grew... I also reported trouble with night glare... I reported more problems with general glare...I thought I was doing the right thing, going to the optician, reporting these symptoms; I was having problems with night driving and the headlight glare and so on so...I said I used to be fine with it but I'm not anymore...So the list was growing, but he didn't write a thing down...In about March 2002 I fell over...I fell again, and this time I fell and I smashed me face and did myself quite a big injury and I went to [named chain of optometrists]...I explained all these symptoms to them...Then she [different optometrist] examined my eyes and she said there was nothing the matter with them...when I came out of [chain of optometrists] in 2002 when she told me, she'd explained away all me symptoms... I came out and I said to myself, I actually gave myself a talking to and said, you're getting older, you're going to have to learn to accept that this is what ageing is, is like. Why do you expect to have young eyes all the time? (P06)

It is worthy of note that the quote above comes from a patient (P06) who reported that her father and grandmother were blind from glaucoma and that in 1992 her optometrist noted an optic disc haemorrhage in her right eye.

I'm the third generation in a row to go blind with this now... I knew glaucoma was a slow advancing disease and I'd been going to the Optometrist since I was 8 ...therefore in all those years I expected them to have noticed something (P06)

Asked when and how her glaucoma was detected she states:

Late on, when $95 \%$ of the optic nerve had been destroyed, in both eyes... In 2003, they [optometrist] did the visual fields and they did the photograph of the retina...they said they were referring me to a doctor because the back of my eyes was a bit of a mess...there's been a massive collapse of the visual fields. (P06)

\section{Service delay}

Patients' referral pathways from optometry detection to specialised eye service appointment varied by location. In Scotland, participants reported being referred directly to specialist services by their optometrist, while in England, referral from optometry to hospital eye services, as reported by all participants, had to be via their GP.

Two participants (P09 and P04) were initially referred to cataract clinics before being redirected, with minimal delay, to specialist glaucoma services. Waiting times for the initial cataract appointments were reported as 2 weeks and 5 months, respectively. One patient reported having ocular hypotensive therapy initiated at the cataract clinic.

\section{...she [ophthalmologist at cataract clinic] said that I [had] severe glaucoma...she did say that if the pressure hadn't come down [with ocular hypotensive therapy] I wouldn't have been able to go home...I was in from 12.30 until about 4 o'clock. (P04)}

For other participants, waiting times between optometry referral and attendance at specialist glaucoma clinics varied from a couple of days to 4 months. However, it is not possible to ascertain from these data whether waiting times reflected the availability of specialist appointments per se (eg, minimum waiting time for all referrals) or the details of the referral (eg, the level of urgency communicated by optometrists or GPs).

So when I went to my GP, which was very quickly after that eye examination within 3 or 4 days. He remarked how he was surprised to see me so quickly and he referred me to the hospital... with the glaucoma specialist but I was marked in a 'no urgency' level so it was actually 15 weeks when I saw somebody. (P06)

Summary of results

The data suggest that late detection of glaucoma can result from delays at the patient level but these findings, although based on a small sample, suggest that delays also occurred at the healthcare provider (system) level both in terms of accuracy of case detection and timely referral.

\section{DISCUSSION}

By exploring accounts of the pathways to detection for patients diagnosed with glaucoma at a late stage, this study makes an important contribution to the evidence on late presentation. Other studies have suggested that late presentation is linked to socioeconomic deprivation and frequency of attending for sight tests in community optometry (ie, patient attributes). ${ }^{4}$ One interpretation of this published evidence is that increased patient awareness and education programmes might encourage earlier detection. Also interventions to break down barriers to access to optometry services at the patient level in areas with high deprivation are being tested. ${ }^{12}$

However, this study highlights that it may be appropriate to design interventions to change behaviour not (only) at the patient level but at the healthcare provider levels (healthcare professionals and referral systems). For example, simple decision support tools, alerts and prompts (delivered as leaflets, wall stickers or through computer systems) could be designed to support optometrists' and GPs' decision making.

Technology could help improve detection. The use of automated optic nerve and retinal nerve fibre layer imaging, particularly in eyes with low intraocular pressure where the glaucoma may be missed, could improve timely glaucoma detection. However, the equipment is costly and there is considerable uncertainty about its place in eye services. A large multicentre comparative study of these imaging technologies for the diagnosis of glaucoma is ongoing and will report in $2015 .^{13}$

\section{Implications for policy}

Strategies to implement the National Institute of Health and Care Excellence guidelines for glaucoma treatment ${ }^{14}$ may raise awareness and knowledge of glaucoma at both the patient and healthcare provider levels. In particular, inclusion of perimetry and optic nerve assessment as part of the optometry test schedule is likely to improve the timeliness and accuracy of referral.

In England, the role of GPs as gatekeepers of care means the referral pathway goes through a generalist (GP) instead of from 'specialist in eyes' (optometry) to 'specialist in eyes' (ophthalmology). While referral refinement systems are in place across the UK with direct referral from optometrists to a specialist eye service, patients' narratives (eg, table 2) indicated there was considerable variation in the post-detection pathway. Consideration should be given to streamlining and standardising this process. 


\section{Strengths and limitations of the study}

The qualitative design is a strength of this exploratory study as it provided vivid, compelling and rich accounts of patients' experiences and new insights into the reality of diagnostic delay.

The small sample size reflected the exploratory nature of the study. The sample was not selected to be statistically representative and we do not claim that our findings are generalisable. The participants' accounts suggest that they may have either open-angle glaucoma or angle-closure glaucoma but all had advanced glaucoma which could have been avoided by early detection and treatment.

\section{CONCLUSION}

The study findings are presented at a time when the main detection pathway issue for glaucoma being addressed in the ophthalmology literature is the significant burden of over-referral of glaucoma suspects to hospital eye services (a large proportion of which are false positives). ${ }^{15}$

In contrast, patients such as those included in this study, who reported attending regular eye tests, but who experienced detection delay and first presented at hospital eye services with advanced glaucoma, represent false negatives. Such patients have been denied the opportunity to access early diagnosis and treatment and thus to limit the extent of their visual impairment. We suggest that attempts to address the significant burden of false positives, by refining glaucoma detection and referral by optometrists, ${ }^{15}$ must also focus on the issue of false negatives and on reducing missed detection and service delays.

Acknowledgements We thank Marion Campbell, Craig Ramsay, Susan Campbell and Jemaima CheHamzah from the Glaucoma screening Platform Study research group for their contribution to the development of the larger study and their guidance throughout its conduct.

Contributors At the time of the research MP, JF, AA-B and JB were at the University of Aberdeen Health Services Research Unit. AA-B and NA identified and made the initial contact with patients meeting the eligibility criteria. MP conducted all interviews. MP, JF and JB had full access to all of the data in the study and can take responsibility for the integrity of the data and the accuracy of the data analysis. All authors participated in the interpretation of results, drafting, revision and approval of the final draft. JB is guarantor.

Funding This study is one component of a Medical Research Council funded strategic grant, G0701759: Developing the intervention and outcome components of a proposed randomised controlled trial of a national screening programme for open-angle glaucoma. The Health Services Research Unit receives a core grant from the Chief Scientist Office of the Scottish Government Health Directorates. All research was conducted independently of the funders.
Competing interests All authors declare that MP, JF, AA-B and JB had support for the submitted work through a Medical Research Council funded strategic grant

Ethics approval North of Scotland research ethics committee.

Provenance and peer review Not commissioned; externally peer reviewed.

Open Access This is an Open Access article distributed in accordance with the Creative Commons Attribution Non Commercial (CC BY-NC 3.0) license, which permits others to distribute, remix, adapt, build upon this work non-commercially, and license their derivative works on different terms, provided the original work is properly cited and the use is non-commercial. See: http://creativecommons.org/ licenses/by-nc/3.0/

\section{REFERENCES}

1 Maier PC, Funk J, Schwarzer G, et al. Treatment of ocular hypertension and open angle glaucoma: meta-analysis of randomised controlled trials. $\mathrm{Br} \mathrm{Med} \mathrm{J}$ 2005;331:134-6.

2 Grant WM, Burke JF. Why do some people go blind from glaucoma? Ophthalmology 1982;89:991-8.

3 Parc $C E$, Johnson $\mathrm{DH}$, Oliver JE, et al. The long-term outcome of glaucoma filtration surgery. Am J Ophthalmol 2001;132:27-35.

$4 \mathrm{Ng}$ WS, Agarwal PK, Sidiki S, et al. The effect of socio-economic deprivation on severity of glaucoma at presentation. Br J Ophthalmol 2010;94:85-7.

5 Fraser $S$, Bunce $C$, Wormald $R$, et al. Deprivation and late presentation of glaucoma: case-control study. Br Med J 2001;322:639-43.

6 Sukumar S, Spencer F, Fenerty $C$, et al. The influence of socioeconomic and clinical factors upon the presenting visual field status of patients with glaucoma. Eye (London) 2009;23:1038-44.

7 Evans J, Ziebland S, McPherson A. Minimizing delays in ovarian cancer diagnosis: an expansion of Andersen's model of 'total patient delay'. Fam Pract 2007;24:48-55

8 Safer MA, Tharps QJ, Jackson TC, et al. Determinants of three stages of delay in seeking care at a medical clinic. Med Care 1979;17:11-29.

9 Andersen BL, Cacioppo JT, Roberts DC. Delay in seeking a cancer diagnosis: delay stages and psychophysiological comparison processes. Br J Soc Psychol 1995:34:33-52.

10 Scott SE, Walter FM, Webster A, et al. The model of pathways to treatment: conceptualisation and integration with existing theory. Br J Health Psychol 2012.

11 Hsiu-Fang H, Shannon SE. Three approaches to qualitative content analysis. Qual Health Res 2005;15.

12 Trudinger $\mathrm{D}$, Niblett $\mathrm{V}$. The barriers and enablers that affect access to primary and secondary eye care services-Hackney site report. London: RNIB, 2012. Report No.: RNIB/CEP/IR/Hackney/01.

13 Comparative study of new imaging technologies for the diagnosis of glaucoma: protocol approved by the Ethics Committee [document on the Internet]. Health Technology Assessment Programme; 2013. http://www.hta.ac.uk/protocols/ 200900220111.pdf (accessed July 2013).

14 National Institute for Health and Clinical Excellence. Glaucoma: Diagnosis and management of chronic open angle glaucoma and ocular hypertension. London: National Institute for Health and Clinical Excellence, 2009. NICE Clinical Guideline 85.

15 Trikha S, Macgregor C, Jeffery M, et al. The Portsmouth-based glaucoma refinement scheme: a role for virtual clinics in the future? Eye 2012;26:1288-94. 